overdose. Check with the relevant authority (details below) if you are uncertain.

- When using the Emergency Department Data Collection remember that diagnoses are often coded by Emergency Department staff as they do their clinical work. Clinical coders are not generally involved in Emergency Department coding. Accuracy of the coding depends on the pressure the staff are under, the ease of use of their Emergency Department information system and other workplace issues that may result in inconsistent or inaccurate codes. For these reasons Emergency Department diagnoses should only be used for coarse analyses and should be considered as indicative only.

- Copies of both the WHO ICD and the clinical modification should be part of your library or you should have access to these, if you are planning anything other than the most superficial analyses.

- You can use the footnotes of graphs or the Methods section of The Health of the People of NSW-Report of the Chief Health Officer to find out what codes to use if you want to conduct analyses of the same disease groups.

\section{SAS EXAMPLES}

Table 1 gives examples of how to create a subset of data in HOIST according to diseases or groupings of diseases. Table 2 lists the commonly used datasets and variables in HOIST that use ICD coding.

The : operator in SAS is useful for selecting ICD groupings. When you put the : after a SAS operator, only the number of characters that you typed are compared. For example, if you type $=$ : ' 038 ' then all codes beginning with 038 are captured, including ' 038 ', '038.4' and '038.42'. It is wise to use the : operator even when you want to select very specific codes also, because sometimes there can be sub-codes that you may not be aware of.

\section{WHERETO GET HELP}

For hospital coding practices contact:

- your local clinical coder or Health Information Manager;

- Senior Clinical Data Consultant, NSW Department of Health by phoning (02) 93919684 ;

- the National Centre for Classification in Health at www.cchs.usyd.edu.au/ncch.

For cause of death coding standards, contact the Vital Statistics Unit of the Australian Bureau of Statistics by phoning 1800620963.

For help with HOIST, contact Alan Willmore, Epidemiology and Surveillance Branch, NSW Department of Health, by emailing awill@doh.health.nsw.gov.au or by phoning (02) 93919226.

\section{ACKNOWLEDGMENTS}

The authors would like to thank staff of the Australian Bureau of Statistics Cause of Death National Project Centre and Joanne Chicco, Senior Clinical Data Consultant, NSW Department of Health for their helpful comments on the draft manuscript.

\title{
TIME TRENDS IN EMERGENCY DEPARTMENT PRESENTATIONS OF CHILDREN WITH ACUTE SEVERE ASTHMA IN NSW
}

\section{Doug Lincoln and David Muscatello \\ Epidemiology and Surveillance Branch NSW Department of Health}

It has been observed that the number of admissions of children to NSW hospitals for asthma increases sharply during the month of February. ${ }^{1}$ Recent anecdotal evidence suggested that this increase occurred again in 2001. Of greater concern, however, is the observation that the number of severe asthma episodes in children also rose sharply. This prompted an investigation to determine whether the reported peak was supported by the data routinely collected by the NSW Department of Health.

The data confirmed the report showing a seasonal increase of asthma presentations, and also highlighted an increasing trend in the proportion of asthma presentations that were severe. The Emergency Department Data Collection (EDDC) was the most up-to-date source of routinely collected data available to investigate this phenomenon. ${ }^{2}$ Despite some shortcomings, the results exemplify the usefulness of the EDDC as a public health surveillance tool. This article describes the methods used and the trends observed using the data from the EDCC.

\section{METHODS}

The EDDC is maintained by the NSW Department of Health and collects information on Emergency Department (ED) activity at 54 hospitals across NSW. It covers most urban hospitals and regional base hospitals. In this article, 'participating EDs' will refer to the EDs of these 54 hospitals. Information collected includes the patient's demographics, primary diagnosis, date and time of presentation, and the clinical response of the participating ED. Information collected at the participating EDs is periodically uploaded to a central data repository held at the NSW Department of Health. At 


\section{FIGURE 1}

SEVERE ASTHMA PRESENTATIONS IN CHILDREN AGED 1-14, NSW, JULY 1996-MAY 2001

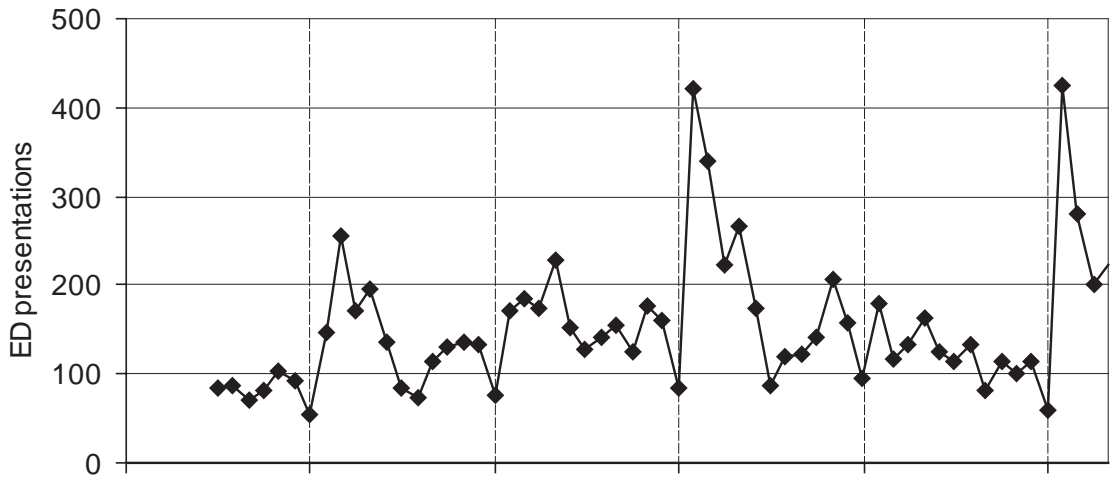

Jan-96 Jan-97 Jan-98 Jan-99 Jan-00 Jan-01

\section{FIGURE 2}

SEVERE ASTHMA PRESENTATIONS AS A PROPORTION OF ALL ASTHMA PRESENTATIONS IN CHILDREN AGED 1-14, NSW, JULY 1996-MAY 2001

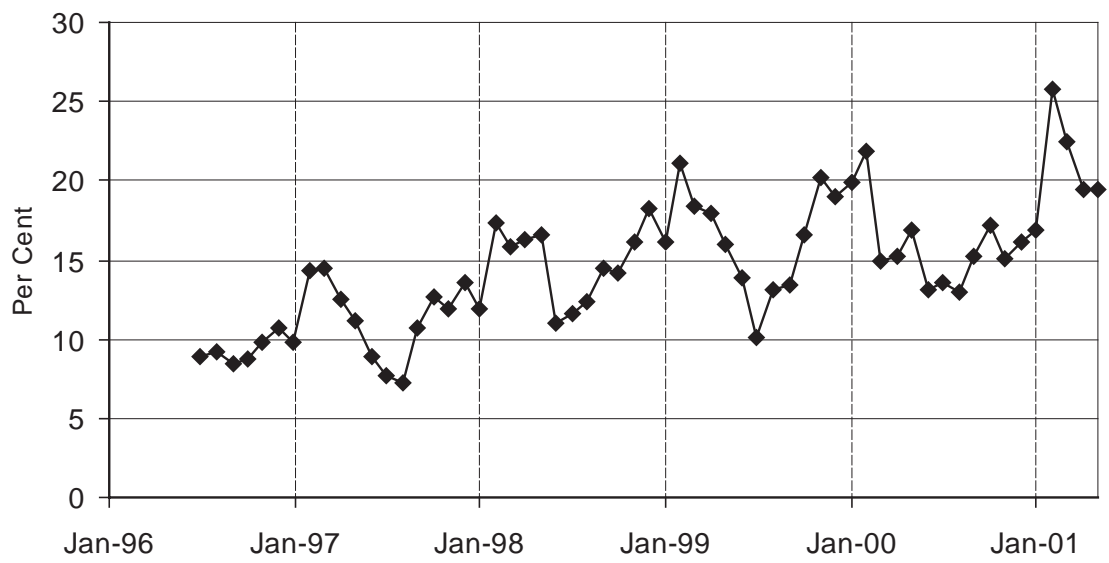

\section{FIGURE 3}

SEVERE ASTHMA PRESENTATIONS BY AGE GROUP, NSW, JULY 1996-MAY 2001

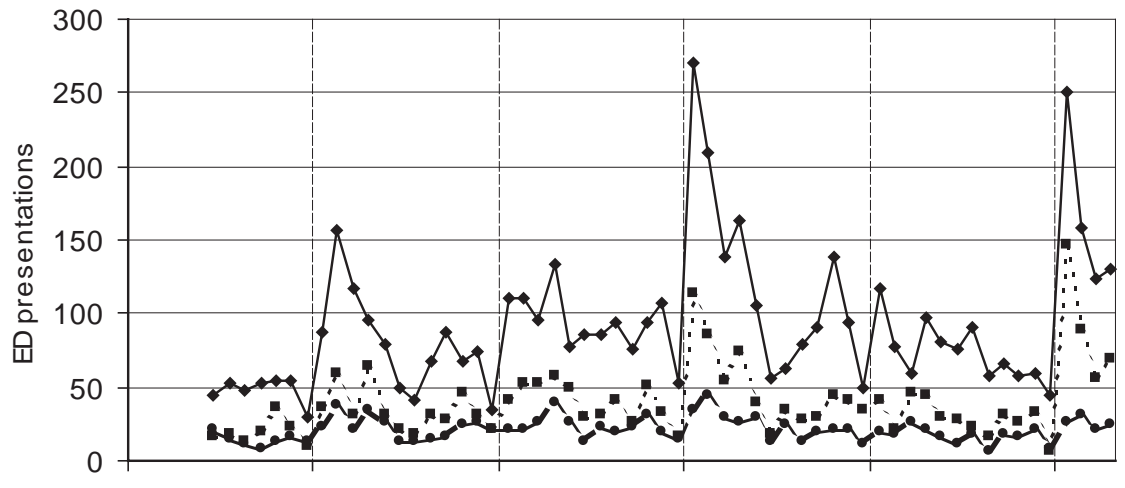

Jan-96 Jan-97 Jan-98 Jan-99 Jan-00 Jan-01

$\longrightarrow-1-4$ yrs $\cdots . .5-9$ years $-\rightarrow--10-14$ yrs 
the time of this investigation, updates were received between every fortnight to one month, which made the EDDC the most up-to-date NSW hospital utilisation information available. The data from six hospitals were removed from the analysis because they had incomplete or non-standard data at the time of this investigation.

We analysed presentations of children aged one to fourteen years of age to participating EDs for the period July 1996 to May 2001. Children aged less than one year were excluded from the analysis, since a diagnosis of asthma can be unclear in this age group. An asthma presentation was a presentation assigned a primary diagnosis of 'asthma' (ICD-9-CM = 493) in the participating ED. ${ }^{3} \mathrm{We}$ used the triage category assigned by the triage nurse on presentation to the participating ED as a proxy for asthma severity. A case of severe asthma was defined to be a presentation assessed as requiring medical attention within 10 minutes according to the Australasian Triage Scale ('resuscitation' or 'emergency' categories in the EDDC). We plotted time series of the number of participating ED presentations for severe asthma to identify any monthly peaks. We also plotted severe asthma as a proportion of all presentations for asthma to assess trends in severity. We also analysed the data by the age groups 1-4, 5-9, and 10-14 years.

\section{RESULTS}

There was a significant peak in the number of presentations of children aged 1-14 years with severe asthma to participating EDs in February 2001. This peak was a sevenfold increase over the number of presentations for severe asthma in the previous month. Similar peaks occurred early in 1997 and 1999 indicating a possible seasonal pattern (Figure 1).

When severe asthma was plotted as a proportion of all asthma presentations, clear peaks were observed in February of each year since 1996. A strong increasing trend was apparent in both the February proportion and the total annual proportion for severe asthma (Figure 2).

The sharp February peaks in presentations for severe asthma were most strongly apparent in children aged 1-4 years, although in February 1999 and 2001 there were also well-defined peaks in presentations for children aged 5-9 years (Figure 3). When the age-specific time series of severe asthma was plotted as a proportion of all presentations for asthma, the two younger age groups showed a pronounced rising trend over the five years of available data. This trend was not apparent in children aged 10-14 years.

\section{DISCUSSION}

The EDDC data supported the anecdotal evidence of a sharp increase in the number of acute cases of severe asthma in children aged 1-14 years in February 2001. This peak was possibly typical of a seasonal pattern of such peaks in the early months of each year, as suggested previously. The peaks occurred mainly in children aged 1-9 years. The proportion of asthma cases that were severe also appears to be increasing over time. This could indicate an increased use of EDs over primary care facilities, a gradual change in triaging patterns in participating EDs, or a true rise in the severity of asthma in children. This rising trend was only apparent in children aged 1-9 years.

The EDDC has several limitations. Firstly, the EDDC includes approximately half of NSW EDs, so the information may not be representative of the whole population of children with asthma in NSW. Also, unlike hospital inpatient information, diagnoses in EDs are not coded by trained clinical coders, but can be entered by a variety of staff in the ED. This means that the standardisation of diagnoses is difficult to maintain. Finally, patterns of ED presentations may be influenced by the availability of general practitioners or other factors influencing ED utilisation. Despite the limitations of the EDDC, the results of this analysis suggest a concerning trend in the incidence of ED presentations for severe asthma in younger children in NSW.

Seasonal peaks in presentations for severe asthma in children in the early part of each year should be anticipated and prevention strategies investigated. Further research into the reasons for these concerning patterns is recommended.

\section{REFERENCES}

1. Sheppard V, Corbett S, Morgan G. A statewide 'outbreak' of asthma in NSW, February 1999. NSW Public Health Bulletin 11(7): 187-189.

2. NSW Department of Health Emergency Department Data Collection (HOIST). Sydney: Epidemiology and Surveillance Branch, NSW Department of Health, 2001.

3. National Coding Centre. The International Statistical Classification of Diseases and Related Health Problems, 9th Revision, Clinical Modification (ICD-9-CM). Sydney: National Coding Centre, 1996. W 